\title{
Fluid-solid coupling dynamic equations considering gas desorption con- traction and coal motion deformations
}

\author{
P. Xu*, Y. Tie ${ }^{* *}$, X.M. Wang*** \\ *Zhengzhou University, Zhengzhou, 450001 Henan, P. R. China, E-mail: plian127@163.com \\ **Zhengzhou University, Zhengzhou, 450001 Henan, P. R. China, E-mail: tieying@zzu.edu.cn \\ ***Chinese Academy of Sciences, Wuhan, Hubei 430071, P. R. China, E-mail: sunwxm@163.com \\ cross $^{\text {ref }}$ http://dx.doi.org/10.5755/j01.mech.23.3.18480
}

\section{Introduction}

There are enormous gas resources in coal mines, and so gas drainage has great important roles before and during coal mine excavation, such as to eliminate gas explosion, gas outburst and other gas accidents during coal mine exploitation, to reduce the air pollution caused by gas emissions, and to adjust and improve the traditional energy structures that are mainly composed of coals and natural gas. To study and understand the mechanism of gas seepage and migration in coals is the key to designing, evaluating and maintaining gas drainage $[1,2]$.

Coals are typical porous media, and so the process of gas drainage would result in two phenomena, to decrease gas pressure, increase the effective stresses of coals, compress coal cracks and pores, and reduce the gas flow channel; to promote coal gas desorption, produce a certain degree of coal matrix shrinkage, and increase the porosity of coals relatively [3]. While gas adsorption also occurs during the process of gas drainage, which always produces certain swelling stresses and then leads to swelling deformation of coals, and so gas seepage is a fluid-solid coupling dynamic process.

In this paper, the fluid-solid coupling model is built that considers gas desorption, motion and deformation of coal solid skeletons, and the model is proved to be reasonable with a computational example of one coal mine in P. R. China.

\section{Dynamic constitutive fluid-solid coupling model}

Under the action of external forces, the coal solid skeletons would produce supporting roles, and the effective stresses are valued as the ratio of supporting forces produced in the solid skeletons to the cross section area. Coal gas is divided into absorbed gas and free gas based on the existing status, and the former occupies over $90 \%$ of the total content. The coals have large specific surface area, so the process of gas absorption belongs to physical absorption, and the absorbed gas would change to free gas under the action of disturbance of temperature, pressure and in-situ stress [4]. Gas drainage from coal bed is actually a reverse process of adsorption expansion, which is also shrinkage desorption of coal matrix, and so the expression of gas drainage is a back-analysis of adsorption-swelling process. The gas seepage process in coals is usually treated as isothermal, so the influence of disturbance of temperature on gas seepage is ignored in relative studies of gas drainage.

\subsection{Moving adaptability equations of coals}

Coals are simplified as homogeneous media and gas as ideal fluid, and the moving adaptability equations of coals include 4 parts as follows [5].

\subsubsection{Effective stresses}

The coals are composed of solid skeletons of molecular-scale particles and inner pores, so the coals that contain gas can be considered as porous media, and the effective stresses of the solid skeletons can be directly depicted as Terzaghi principle of effective stresses:

$$
\sigma_{i j}=\sigma_{i j}^{\prime}+\alpha p \delta_{i j}
$$

where $\sigma_{i j}$ are the total stresses, $\sigma_{i j}^{\prime}$ are effective stresses, $\alpha$ is Biot's parameter, $p$ is gas pressure, and $\delta_{i j}$ is Kronecker's delta symbol, $\delta_{i j}=1$ when $i=j$ while $\delta_{i j}=0$ when $i \neq j$.

\subsubsection{Constitutive equations}

Similar to the conventional elastic materials, the physical equations of coal solid skeletons can be given based on elastic theory:

$$
\sigma_{i j}=2 G \varepsilon_{i j}+\lambda \varepsilon_{v} \delta_{i j},
$$

where $\varepsilon_{i j}$ are strains of coal solid skeletons, $\varepsilon_{v}$ is volumetric strain of coal solid skeletons, $\lambda$ and $G$ are Lamé elastic constants of coal solid skeletons, $\lambda=\frac{\mu E}{(1+\mu)(1-2 \mu)}$ and $G=\frac{E}{2(1+\mu)}, E$ and $\mu$ are elastic modulus and Poisson's ratio of coal solid skeletons respectively.

The geometric equations of coal solid skeletons can also be given directly based on elastic theory:

$$
\varepsilon_{i j}=\frac{1}{2}\left(u_{i, j}+u_{j, i}\right)
$$

where $u$ is the displacement of the coal solid skeletons.

The displacement $u$ of coal solid skeletons is a dynamic variable and includes parameters of space and time, so $u$ has the expression of $u=u(x, y, z, t)$, and the equations of dynamic equilibrium of coal solid skeletons are: 


$$
\sigma_{i j, j}^{\prime}+F_{i}=\rho \ddot{u}_{i}+\rho_{g} \ddot{w}_{i},
$$

where $w$ is relative displacement of coal solid skeletons comparative to gas, $F$ is body stress, $\rho$ is the total physical density of coals and is the sum of solid skeletons and gas, $\rho=\varphi \rho_{s}+(1-\varphi) \rho_{g}, \varphi$ is porosity, and $\rho_{s}$ and $\rho_{g}$ are the densities of coal solid skeletons and gas respectively.

Substituting Eqs. (1) and (2) into Eq. (4), the equations of dynamic equilibrium are

$$
(\lambda+G) u_{j, j i}+G u_{i, j j}+F_{i}=\alpha p_{, i}+\rho \ddot{u}_{i}+\rho_{g} \ddot{w}_{i},
$$

According to the relevant expressions of $u_{j, j}=\nabla \cdot \boldsymbol{u}$ and $u_{i, j j}=\nabla^{2} u_{i}$, Eq. (5) can be rewritten in vectors:

$$
(\lambda+G) \nabla(\nabla \cdot \boldsymbol{u})+G \nabla^{2} \boldsymbol{u}+\boldsymbol{F}=\alpha \nabla p+\rho \ddot{\boldsymbol{u}}+\rho_{g} \ddot{\boldsymbol{w}}
$$

2.2. Gas seepage equations that consider deformation of coal solid skeletons

\subsubsection{Dynamic porosity}

The pores such as holes, cracks and fissures in coals provide pathways for gas migration, so the coal porosity is one of the key physical parameters to study the characteristics of gas transportation. Porosity $\varphi$ is the ratio of volume of pores to the total volume of coals, it changes with the deformation of coals, so it is a relative variable influenced by the loads that applied on the coals, and the equation of $\varphi$ during the isothermal process is given as follows [6]:

$$
\varphi=1-\frac{1-\varphi_{0}}{1+\varepsilon_{v}}\left(1-\frac{\Delta p}{K_{s}}\right),
$$

where $\varphi_{0}$ is the initial porosity of coals under certain fiducial temperature and without any outer loads, $K_{s}$ is bulk modulus of soil solid skeletons and $K_{s}=E / 3(1-2 \mu)$, and $\Delta p$ is the changed gas pressure from the initial gas pressure $p_{0}$ and $\Delta p=p-p_{0}$.

\subsubsection{Dynamic permeability}

Similar to porosity of coals, permeability of coals is also a dynamic parameter that changed with porosity, and permeability $k$ can be simplified as follows based on Krozeny-Carman equation in fluid mechanics [7]:

$$
k=\frac{k_{0}}{1+\varepsilon_{v}}\left(1+\frac{\varepsilon_{v}}{\varphi_{0}}+\frac{1-\varphi_{0}}{\varphi_{0}} \frac{\Delta p}{K_{s}}\right)^{3},
$$

where $k_{0}$ is the initial permeability of coals under certain fiducial temperature and without any outer loads.

\subsubsection{Gas flow in coals}

Gas in coals is assumed as ideal fluid, and then gas density and pressure should satisfy with [8]:

$$
\begin{aligned}
& \rho_{g}=\beta p, \\
& \beta=\frac{M}{R T},
\end{aligned}
$$

where $\beta$ is the compression coefficient of gas, $M$ is the molecular weight of gas, $R$ is ideal gas constant, and $T$ is absolute temperature.

Gas content $Q$ is composed of free gas content $Q_{f}$ and absorbed gas content $Q_{a}$ :

$$
\begin{aligned}
& Q=Q_{f}+Q_{a}, \\
& Q_{f}=\varphi \rho_{g}, \\
& Q_{a}=\frac{\rho_{a} \rho_{s} V_{L} p}{P_{L}+p},
\end{aligned}
$$

where $Q_{f}$ and $Q_{a}$ are calculated with Langmuir's formula, $P_{L}$ is Langmuir's pressure, under which the absorption capacity of gas can reach $50 \%$ of the maximum value, $V_{L}$ is Langmuir volume that reflects the maximum adsorption capacity, $\rho_{a}$ is gas density that measured under standard conditions and $\rho_{a}=\beta p_{a}$ that is obtained from Eq. (9), and $p_{a}$ is the standard atmospheric pressure with constant value of $1.013 * 10^{5} \mathrm{~Pa}$.

Substituting Eqs. (9), (12) and (13) into Eq. (11), the total gas content is obtained:

$$
Q=\beta\left(\varphi+\frac{\rho_{s} p_{a} V_{L}}{P_{L}+p}\right) p .
$$

Under pressure gradient $\nabla p$, the process of gas flow in coals satisfies with Darcy's law

$$
\boldsymbol{q}=-\frac{k}{\eta} \nabla p
$$

where $\boldsymbol{q}$ is the velocity vector of gas seepage and $\eta$ is dynamic viscosity of gas.

Gas seepage in coals meets with quality conservation law, as to the unit volume of coals, the seepage equation is:

$$
\frac{\partial Q}{\partial t}+\nabla \cdot\left(\rho_{g} \boldsymbol{q}\right)=0
$$

\subsubsection{Volumetric strain of coals}

Deformation of coals caused by gas pressure and gas desorption is considered, and the volumetric strain $\varepsilon_{v}$ of coal solid skeletons is composed of 3 parts:

$$
\varepsilon_{v}=\varepsilon_{v}^{s}+\varepsilon_{v 1}^{g}+\varepsilon_{v 2}^{g},
$$


where $\varepsilon_{v}^{s}$ is the volumetric strain that caused by the deformation of solid skeletons, and it can be calculated from Eq. (3) as follows:

$$
\varepsilon_{v}^{s}=u_{i, i}
$$

The volumetric strain $\varepsilon_{v 1}^{g}$ is caused by gas pressure increment $\Delta p$, and can be calculated as follows:

$$
\varepsilon_{v 1}^{g}=-\frac{\Delta p}{K_{s}}
$$

and the volumetric strain $\varepsilon_{v 2}^{g}$ is caused by gas desorption, and it can be calculated as follows [9]:

$$
\varepsilon_{v 2}^{g}=\alpha_{g}\left(\frac{p}{P_{L}+p}-\frac{p_{0}}{P_{L}+p_{0}}\right),
$$

where $\alpha_{g}$ is the thermal expansion coefficient of gas adsorption and it is equal to Langmuir's volumetric strain $\varepsilon_{L}$ in numerical value.

And $\varepsilon_{v}$ Eq. (17) is obtained:

$$
\varepsilon_{v}=u_{i, i}-\frac{\Delta p}{K_{s}}+\alpha_{g}\left(\frac{p}{P_{L}+p}-\frac{p_{0}}{P_{L}+p_{0}}\right) .
$$

$$
\begin{gathered}
\beta\left\{\frac{\varphi}{p}+\frac{\rho_{s} p_{a} V_{L} P_{L}}{\left(P_{L}+p\right)^{2}}+\frac{1-\varphi_{0}}{\left(1+\varepsilon_{v}\right) K_{s}}+\frac{1-\varphi}{1+\varepsilon_{v}}\left[\frac{\alpha_{g} P_{L}}{\left(P_{L}+p\right)^{2}}-\frac{1}{K_{s}}\right]\right\} \frac{\partial p^{2}}{\partial t}-\nabla \cdot\left(\beta \frac{k}{\eta} \nabla p^{2}\right)=-2 \rho_{g} \frac{1-\varphi}{1+\varepsilon_{v}} \dot{u}_{i, i}, \\
(\lambda+G) \nabla(\nabla \cdot \boldsymbol{u})+G \nabla^{2} \boldsymbol{u}+\boldsymbol{F}=-\alpha \frac{\eta}{k}(\dot{\boldsymbol{u}}+\dot{\boldsymbol{w}})+\rho \ddot{\boldsymbol{u}}+\rho_{g} \ddot{\boldsymbol{w}}, \\
\beta\left[\frac{\varphi}{p}+\frac{\rho_{s} p_{a} V_{L} P_{L}}{\left(P_{L}+p\right)^{2}}+\frac{\varphi-\varphi_{0}}{\left(1+\varepsilon_{v}\right) K_{s}}\right] \frac{\partial p^{2}}{\partial t}-\nabla \cdot\left(\beta \frac{k}{\eta} \nabla p^{2}\right)=-2 \rho_{g} \frac{1-\varphi}{1+\varepsilon_{v}} \dot{u}_{i, i}, \\
(\lambda+G) \nabla(\nabla \cdot \boldsymbol{u})+G \nabla^{2} \boldsymbol{u}+\boldsymbol{F}=-\alpha \frac{\eta}{k}(\dot{\boldsymbol{u}}+\dot{\boldsymbol{w}}) .
\end{gathered}
$$

\section{Engineering example}

The gas drainage engineering in the $2^{\text {nd }}$ coal bed of one coal mine in Henan province of P. R. China is taken as a computational example. The diameter of the gas drainage hole is $100.0 \mathrm{~mm}$, the coal seam is about $6.0 \mathrm{~m}$

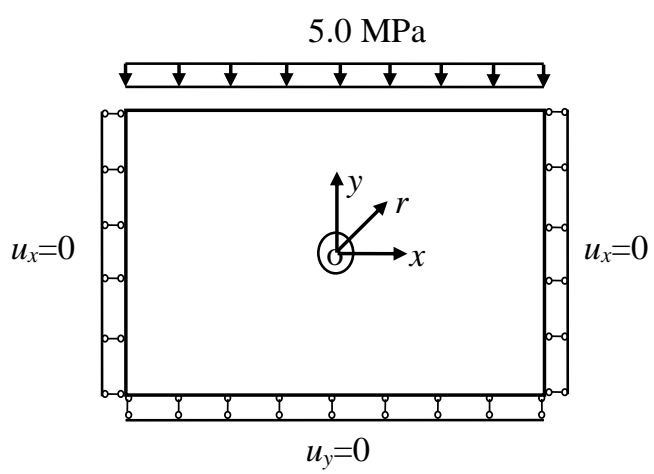

Fig. 1 Simplified 2-dimensional computational model thick, the length of gas drainage hole and coal thickness are all much larger than the diameter of the gas drainage hole, and so the gas drainage process can be simplified as a 2-dimensional plane strain problem.

The computational model is given in Fig. 1, which is a rectangle with length of $100.0 \mathrm{~m}$ and height of $6.0 \mathrm{~m}$, the origin of rectangular coordinate system is located at the center of gas drainage hole, and axis $x$ and $y$ are along horizontal and vertical direction respectively.

\subsection{Initial and boundary conditions}

Displacement constraints are applied in $x$ direction on the left boundary $(x=-50.0 \mathrm{~m})$ and right boundary $(x=50.0 \mathrm{~m})$ and in y direction on the bottom boundary $(y=-$ $-3.0 \mathrm{~m}$ ), and uniform pressure of 5.0 $\mathrm{MPa}$ is applied on the top boundary $(y=3.0 \mathrm{~m})$, which is the pressure caused by the total weights of soils and rocks that just overly on the computational coal bed. The model has no initial dis- 
placements and velocities, that is to say that $u_{i}=0$ and $\dot{u}_{i}=0$ when $t=0$.

The coal roof and floor are all mudstones with negligible permeability, and so the up and bottom boundaries of the computational model are assumed as impermeable layers. The coal bed is horizontally distributed and the length of calculation model is built too long to exceed the influence scope of gas drainage process, and so the left and right boundaries of the computational model are also assumed to have no gas seepage. The outer and inner boundary conditions in Fig. 1 are depicted as follows, $p_{x}=0$ on the up and bottom boundaries, $p_{y}=0$ on the left and right boundaries, $p=p_{w}$ on the inner boundary of the gas drainage hole, in which $p_{w}$ is the gas drainage pressure, and the initial gas pressure of the model is $p_{x}=p_{y}=p_{0}$.

The coals are assumed as homogeneous isotropic media, and the main physical and mechanical parameters of coals and gas of model I are given in Table 1 . As to model II, only $\alpha_{g}$ is valued as 0 and other parameters are valued same to model I.

Table 1

Main physical and mechanical parameters

\begin{tabular}{|c|c|}
\hline Parameter & Value \\
\hline Biot's parameter $\alpha$ & 0.99 \\
\hline Elastic modulus of coals $E, \mathrm{MPa}$ & 9.8 \\
\hline Poisson's ratio of coals $\mu$ & 0.35 \\
\hline Density of coal solid skeletons $\rho_{\mathrm{s}}, \mathrm{kg} / \mathrm{m}^{3}$ & 1250 \\
\hline Density of gas $\rho_{\mathrm{g}}, \mathrm{kg} / \mathrm{m}^{3}$ & 0.714 \\
\hline Dynamic viscosity of gas $\eta, \mathrm{P} \cdot \mathrm{s}$ & $1.64^{*} 10^{-5}$ \\
\hline Langmuir's pressure $P_{L}, \mathrm{MPa}$ & 5.39 \\
\hline Langmuir's volume $V_{L}$ & 0.015 \\
\hline Expansion coefficient of gas adsorption $\alpha_{g}$ & 0.022 \\
\hline Initial gas pressure $p_{0}, \mathrm{MPa}$ & 1.40 \\
\hline Gas drainage pressure $p_{w}, \mathrm{MPa}$ & 0.05 \\
\hline Initial porosity $\varphi_{0}$ & 0.085 \\
\hline Initial permeability $k_{0}, \mathrm{~m}^{2}$ & $7.8^{*} 10^{-17}$ \\
\hline
\end{tabular}

\subsection{Gas seepage calculation}

The curves of radial displacement $u_{r}$ at the inner side of the drainage hole changing with distances from the gas drainage hole center are given in Fig. 2.

Fig. 2 shows that the elastic radial displacement $u_{r}$ of the two computational models of I and II all increase during the gas drainage process, while $u_{r}$ of model $\mathrm{I}$ is larger than model II, that is to say that if the gas desorption shrinkage and moving adaptability are considered, the gas drainage hole would turn to be valid earlier than the traditional calculation model II. And $u_{r}$ of model I reaches $16.2 \mathrm{~mm}$ at $t=50 \mathrm{~d}$, which is about $0.8 \mathrm{~mm}$ larger than that of model II at the same time, and the former is $32.4 \%$ of the original radius of $50 \mathrm{~mm}$.

The line at section of $y=0 \mathrm{~m}$ and $x=0 \sim 50 \mathrm{~m}$ is selected, and the curves of gas pressure $p$ at $t=50 d$ changing with distances from the drainage hole center are given as shown in Fig. 3.

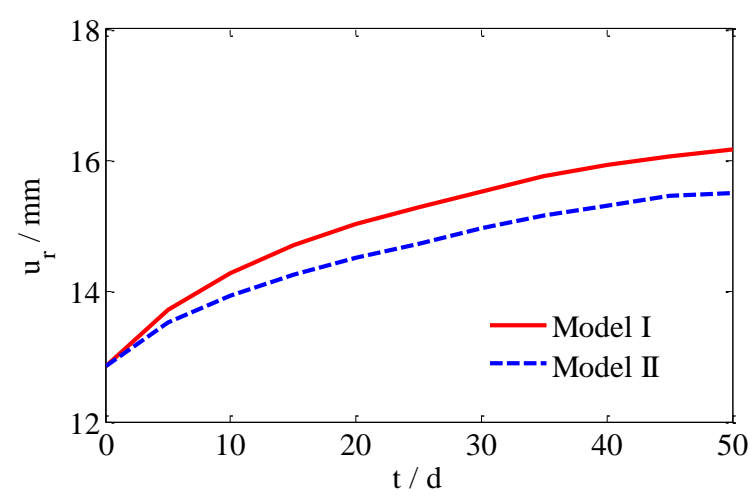

Fig. 2 Curves of radial displacements at the gas-drainage hole

Fig. 3 shows that the gas pressure of coal seam calculated by the model I is about $7.6 \%$ less than model II, and this phenomenon can be interpreted by the mechanism of gas extraction as follows: during the gas drainage process, the solid skeletons move towards the drainage hole caused by the gas flows, some expansion deformation of gas flow would occur, which can also be found in Fig. 2, desorption and contraction of coal solid skeletons would also occur, and then coal porosity increase and gas permeability is improved correspondingly. And at the actual gas drainage process of this mine in Henan Province of P. R. China, the drained gas contents usually increase about $8 \%$ after the beginning drainage for 10 days, which just meets with the calculation results of model I. The calculated gas pressure of model I is less than that of model II near the gas drainage hole at the same time, while they turn to be same at farer place where the distance is larger than $40 \mathrm{~m}$ from the drainage hole center, where the gas pressure is just equal to the original gas pressure of $1.40 \mathrm{MPa}$ that is given in Table 1.

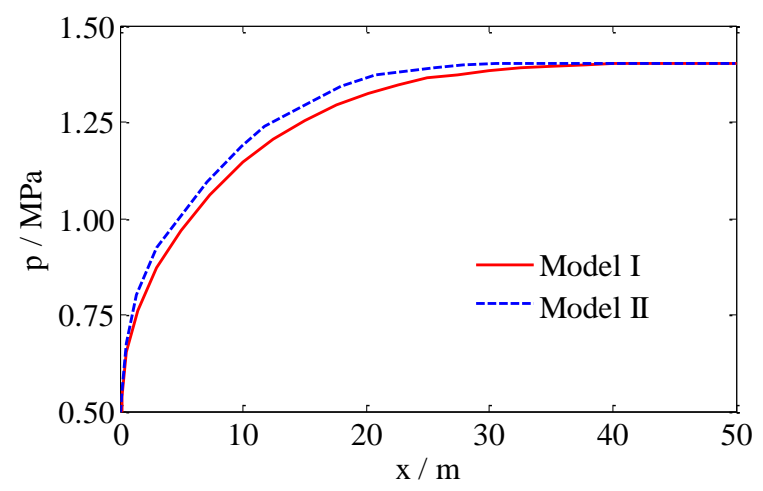

Fig. 3 Comparison curves of gas pressure changing with distances

Figs. 2 and 3 show that model I is more reasonable than the traditional model II, and so model I is selected for the further analyses about gas pressure and gas permeability during the gas drainage process in this paper.

The drainage time of $t=50 \mathrm{~d}$ is selected, and cloud figure of gas pressure around the gas drainage hole in the location of $r \leq 0.8 \mathrm{~m}$ is given in Fig. 4 .

Fig. 4 shows that the shapes of gas pressure are regular circular rings and change from small value at the inside locations near the gas drainage hole to large value at the outside locations, which meets with the fact that the 
more the gas are drained out and the less the gas pressure would be.

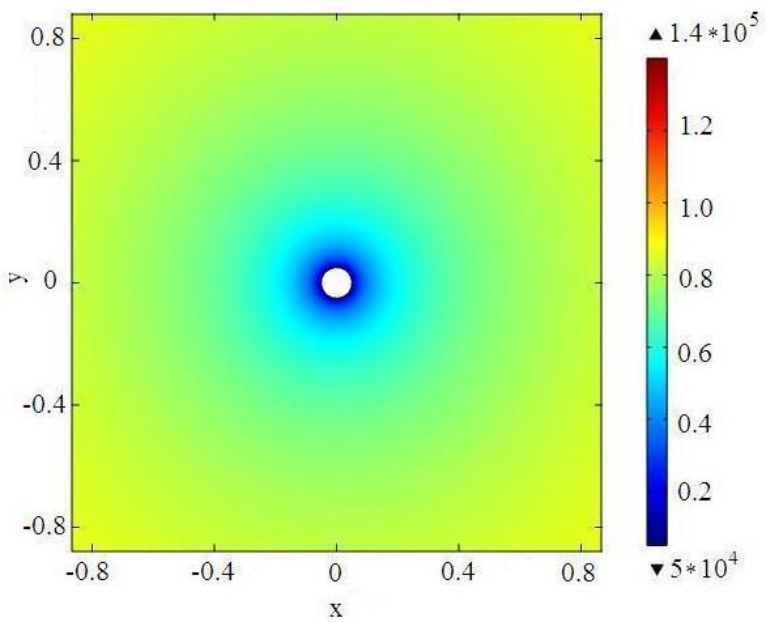

Fig. 4 Cloud figure of gas pressure around the gas drainage hole $(t=50 d)$

Curves of gas pressure curves at different position $(x=1,5,10$ and $15 \mathrm{~m}$ ) changing with drainage time $t$ and at different drainage time ( $t=10,50,100$ and $150 d$ ) changing with distance $x$ are given in Figs. 5 and 6 respectively.

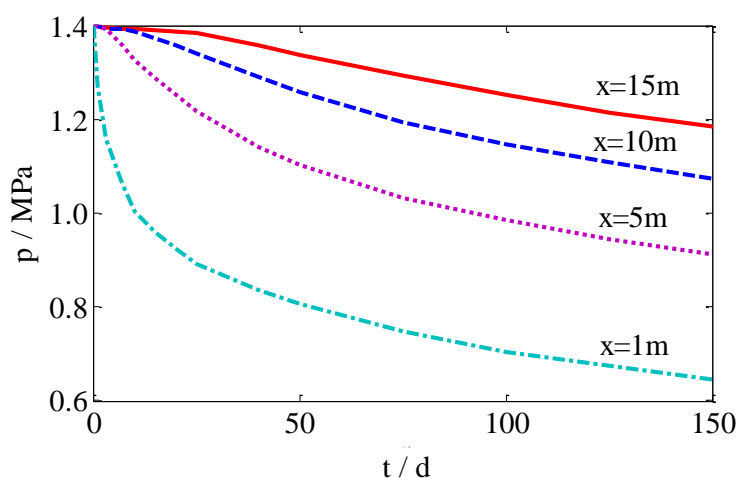

Fig. 5 Gas pressure curves changing with draining time at different positions

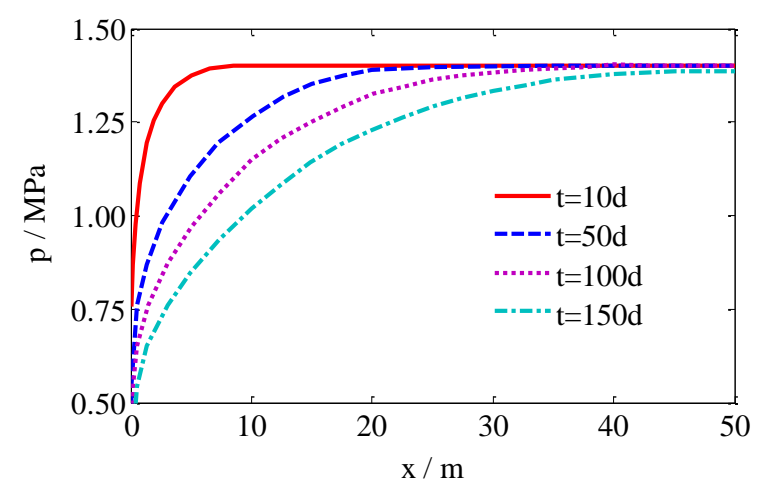

Fig. 6 Curves of gas pressure changing with distance at different drainage time

Fig. 5 shows that gas pressure at positions near drainage hole is less than the farer position, and the gas pressure decreases with the ratio of about $0.0012 \mathrm{MPa} / d$.

Fig. 6 shows that gas pressure in the farer place is mainly influenced by the gas drainage date, the place of $x=20 \mathrm{~m}$ is taken for example, the reduced gas pressures are
0, 0, 0.11 and 0.25 MPa for $t=10,50,100$ and $150 d$ respectively, and these principles and characteristics meet with the relative curves in Ref. [4]. Gas pressure would reach the initial value at the positions farer from the gas drainage hole center such as $x=40 \mathrm{~m}$.

Similar to Fig. 4, the drainage time of $t=50 \mathrm{~d}$ is also selected, and cloud figure of gas permeability at the scope of $r \leq 0.8 \mathrm{~m}$ is given in Fig. 7 .

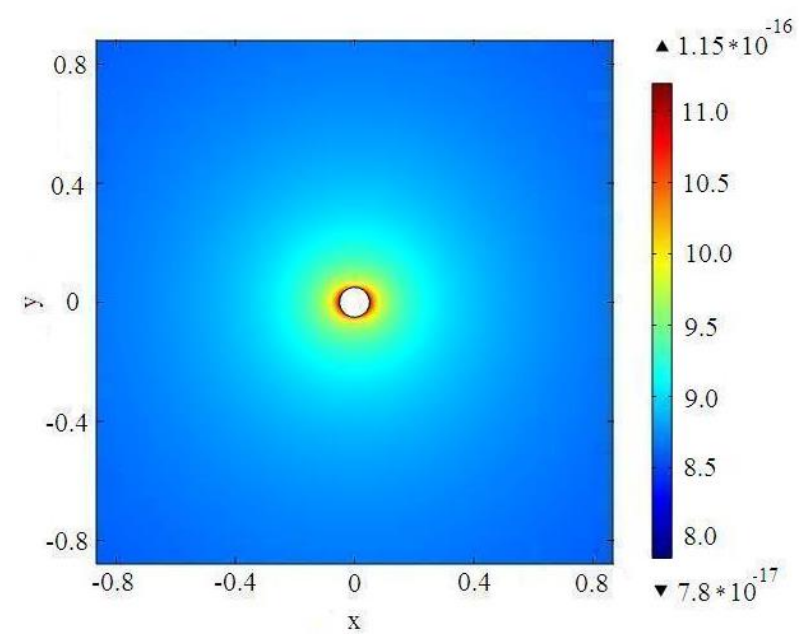

Fig. 7 Cloud figure of gas permeability around the gasdrainage hole $(t=50 \mathrm{~d})$

Fig. 7 shows that when the gas near the drainage hole is drained in large amount, the gas permeability increases obviously, and this phenomenon can be explained as follows. When gas is drained out, coal solid skeletons have radial displacement towards the gas drainage hole as shown in Fig. 2, and so coal porosity and gas permeability near the drainage hole all increase. The maximum permeability is about $1.15^{*} 10^{-16} \mathrm{~m}^{2}$ near the drainage hole, which is about 1.5 times of the initial permeability of $7.8 * 10^{-17}$ $\mathrm{m}^{2}$ that is given in Table 1 .

Similar to Figs. 5 and 6, curves of coal permeability at different position $(x=1,5,10$ and $15 \mathrm{~m})$ changing with drainage time $t$ and at different drainage time $(t=10$, 50,100 and $150 d$ ) changing with distance $x$ are given in Figs. 8 and 9 respectively.

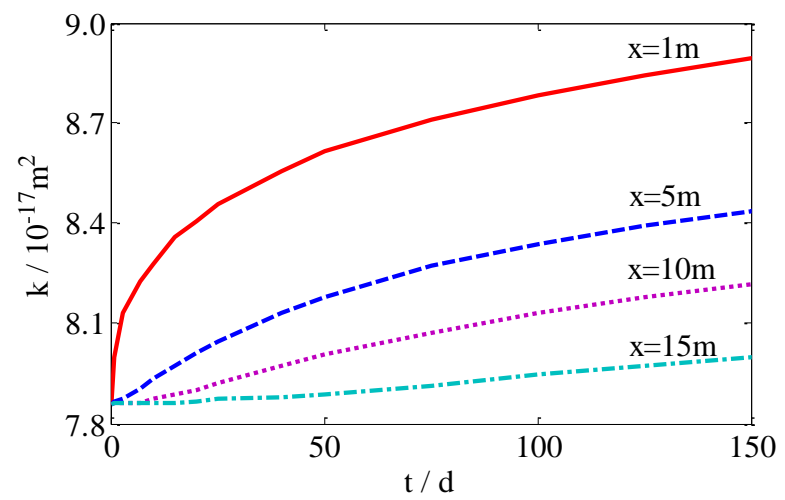

Fig. 8 Curves of permeability changing with draining time at different positions

Figs. 8 and 9 show that gas permeability increases obviously at the positions near the drainage hole, the maximum increasing permeability ratio is $0.0022 * 10^{-17} \mathrm{~m}^{2} / d$, and gas permeability would reach the initial value at the positions farer from the gas drainage hole center such as 
$x=20 \mathrm{~m}$, and this phenomenon is similar to gas pressure shown in Figs. 5 and 6.

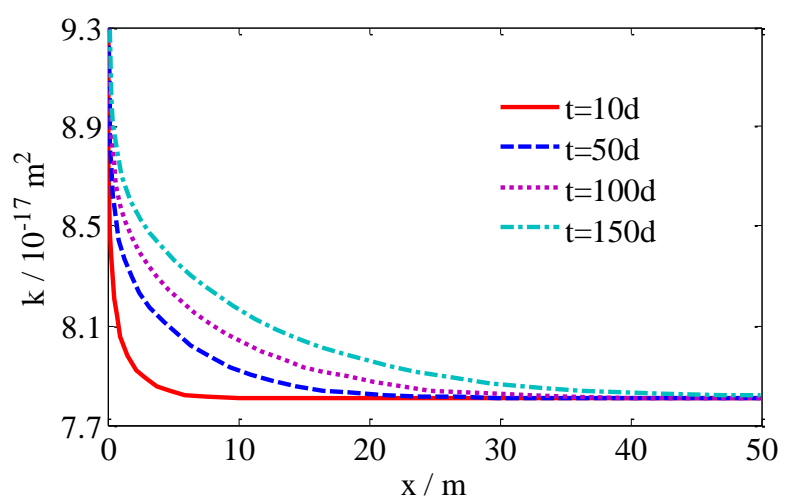

Fig. 9 Curves of permeability changing with distances from the hole at different positions

\section{Conclusions}

1. Coals that contain gas are assumed as isotropic and homogeneous elastic media, the shrinkage deformation caused by gas desorption and motion deformation caused by gas flow are considered, and the fluid-solid coupling dynamic equations about gas drainage are built, which are mainly composed of gas seepage, motion of the coal skeletons and shrinkage deformation of coals. And the model built in this paper is proved to be more reasonable than the traditional model that ignores gas desorption and shrinkage.

2. The radial displacement around the gas drainage hole reaches $16.2 \mathrm{~mm}$ at $t=50 \mathrm{~d}$, which is $32.4 \%$ of the original radius of $50 \mathrm{~mm}$.

3. Gas pressure and permeability that influenced by the gas drainage process are mainly focused at the locations near the gas drainage hole, and they are equal to the initial values at the farer locations.

\section{Acknowledgements}

This work is supported by National Natural Science Foundation of China (Grant No. 51278467), Program for Science and Technology Innovation Talents in Universities of Henan Province (Grant No. 14HASTIT050) and Special Scientific Research Foundation for Young Teachers of Zhengzhou University (Grant No. 1421323078).

\section{References}

1. Bustin, R.M.; Clarkson, C.R. 1998. Geological controls on coalbed methane reservoir capacity and gas content, International Journal of Coal Geology, 38 (12), 3-26.

http://dx.doi.org/10.1016/S0166-5162(98)00030-5. http://www.sciencedirect.com/science/article/pii/S0166 516298000305.

2. Xu, P.; Li, X.C.; Fang, Z.M.; et al. 2012. Application of quantification theory to predict coal methane content, Disaster Advances, 5(4), 1609-1614.

3. Levine, J.R. 1996. Model study of the influence of matrix shrinkage on absolute permeability of coal bed reservoirs, Geological Society, London, Special Publications, 109(1): 197-212.

http://dx.doi.org/10.1144/GSL.SP.1996.109.01.14.

4. Si, H.; Guo, T.; Li, X.H. 2011. Analysis and numerical simulation of fluid-structure coupling of gas drainage from boreholes, Journal of Chongqing University, 34(11): 105-110. (in Chinese)

5. Biot, M.A. 1956. Theory of propagation of elastic waves in a fluid-saturated porous solid. I. Lowfrequency range. The Journal of the acoustical Society of America, 28(2), 168-178.

http://dx.doi.org/10.1121/1.1908239.

6. In, G.Z.; Wang, D.K.; Zhang, D.M.; et al. 2008, Solid-gas coupling dynamic model and numerical simulation of coal containing gas, Chinese Journal of Geotechnical Engineering, 10(4): 1430-1435. (in Chinese)

7. Yang, T.H.; Chen, S.K.; Zhu, W.C.; et al. 2010. Coupled model of gas-solid in coal seams based on dynamic process of pressure relief and gas drainage, Rock and Soil Mechanics, 31(7): 2247-2252. (in Chinese)

8. Cooper, J.W.; Wang, X.; Mohanty, K.K. 1999. NonDarcy-flow studies in anisotropic porous media, SPE Journal, 4(4), 334-341. http://dx.doi.org/10.2118/57755-PA.

9. Liu, Y.B.; Cao, S.S.; Li, Y.; et al. 2010. Experimental study of swelling deformation effect of coal induced by gas adsorption[J]. Chinese Journal of Rock Mechanics and Engineering, 2010, 29(12): 2484-2491. (in Chinese).

\section{P. Xu, Y. Tie, W.M. Wang}

\section{FLUID-SOLID COUPLING DYNAMIC EQUATIONS CONSIDERING GAS DESORPTION CONTRACTION AND COAL MOTION DEFORMATIONS}

\section{S u m m a r y}

The coals are assumed as isotropic homogeneous saturated porous media, dynamic characteristics of porosity and permeability of coals and coal deformation caused by gas desorption are all considered, gas seepage equations about porosity, permeability and desorption contraction deformation are built based on Darcy's law, and fluid-solid coupling equations for gas seepage are finally built, which are composed of the above-mentioned dynamic balance differential equations and gas seepage equations. In the end, one horizontal gas drainage hole is taken as a computational example, the gas pressures calculated by the new model built in this paper are compared with those by the normal model that does not include gas adsorption and motion deformation of coals, the new model is proved to be reasonable based on the mechanism of desorption contraction and seepage, and the characteristics of permeability, gas pressure and radial displacements around the hole are analyzed based on the new model.

Keywords: gas drainage, gas desorption, gas seepage, motion deformation, fluid-solid coupling equations.

Received March 08, 2016 Accepted June 08, 2017 